\title{
Effective Capacity of a Correlated Nakagami-m Fading Channel
}

\author{
Qing Wang, Dapeng Wu and Pingyi Fan
}

\begin{abstract}
The grail of next-generation wireless networks is providing real-time services for delay-sensitive applications, which require that the wireless networks provide QoS guarantees. The effective capacity (EC) proposed by Wu and Negi provides a powerful tool for design of QoS provisioning mechanisms. In this paper, we intend to generalize their formula for the effective capacity of a correlated Rayleigh fading channel; specifically, we derive a closed form approximate EC formula for a special correlated Nakagami-m fading channel, for which the inverse of the correlation coefficient matrix is tridiagonal. To verify its accuracy via simulation, we develop a Green-matrix based approach, which allows us to analytically obtain the effective capacity (given the joint probability density function of a correlated Nakagami-m fading channel) while being able to simulate the corresponding channel gain process. Simulation results show that our EC formula is accurate. Furthermore, to facilitate the application of the EC theory to the design of practical QoS provisioning mechanisms, we propose a simple algorithm for estimating the EC of an arbitrary correlated Nakagami-m fading channel, given channel measurements; simulation results demonstrate the accuracy of our proposed EC estimation algorithm, showing its suitability in practice.
\end{abstract}

\section{Index Terms}

Effective capacity, correlated Nakagami-m fading, Rayleigh fading, QoS provisioning.

\section{INTRODUCTION}

The next-generation wireless networks (e.g., 4G cellular networks, WiMax, IEEE 802.11n, and nextgeneration mobile ad hoc networks) are targeted at supporting various applications such as voice, data, and multimedia over packet-switched networks. The grail of these networks is providing real-time services for delay-sensitive applications. Delay sensitive applications such as voice-over-IP, interactive video, mobile $\mathrm{TV}$, and interactive gaming, require that the wireless networks provide quality of service (QoS) guarantees, e.g., guarantees on data rate, delay bound, and delay bound violation probability.

The general objective of this paper is to explore the performance limits in delay-constrained wireless networks. To address this, one can take the following approaches. The first approach is network calculus (a

Qing Wang and Pingyi Fan are with Tsinghua University, Beijing, P. R. China. Dapeng Wu is with Department of Electrical and Computer Engineering, University of Florida, Gainesville, FL 32611. Correspondence author: Prof. Dapeng Wu, wu@ece.ufl.edu, http://www.wu.ece.ufl.edu. 
bounding approach) [1], [2]; in this approach, the number of parameters needed to describe the performance measures is small but the obtained performance measures are not accurate due to simplification in source/channel characterization. The second approach is parametric stochastic modeling [3], [4]. It models the statistics (e.g., marginal probability density functions, transition probabilities, or auto-correlation functions [5]) of stochastic processes for the arrivals and departures in the system. Markov modulated Poisson processes (MMPP) [6] are such models. To achieve accurate performance prediction, the number of parameters in the stochastic model is usually large, resulting in high complexity of estimating model parameters from real traffic/channel measurements. This also leads to high complexity in queueing analysis. The third approach is large deviations [7], [8]. This approach is simple in terms of the number of parameters needed for quantifying performance; the performance measure obtained is proven to be accurate asymptotically, i.e., the performance measure is accurate in the large delay regime but not in the small delay regime. However, surprisingly, it is showed that the performance measure obtained by large deviation techniques is accurate even in the small delay regime (e.g., from $50 \mathrm{~ms}$ to $500 \mathrm{~ms}$ as in Fig. 1) for Rayleigh/Rician/Nakagami-m fading with a practical Doppler spectrum and the velocity of a mobile terminal ranging from $0.041 \mathrm{~km} / \mathrm{h}$ to $204 \mathrm{~km} / \mathrm{h}$ [9], [10]. ${ }^{1}$ This important finding opens a new avenue for QoS provisioning in wireless networks [12]. Using large deviations theory, the effective capacity approach [9], [10] was developed, which has been applied in multiple-input-multiple-output (MIMO) systems [13], adaptive-modulation-and-coding and power control [14], amplify-and-forward and decode-and-forward relay networks (cooperative diversity) [15], resource allocation [16], multi-hop delay performance in wireless mesh networks [17], joint design of video compression, link layer and physical layer [18], and study of joint impact of bandwidth, power, and code-rate on the effective capacity [19].

The effective capacity (EC) [10] provides a powerful tool for exploring the performance limits in delayconstrained wireless networks, and designing QoS provisioning mechanisms in wireless networks. The authors [10] derived a formula for the effective capacity of a Rayleigh fading channel with arbitrary Doppler spectrum [20]. In this paper, we intend to generalize their formula for the effective capacity of a correlated Rayleigh fading channel; specifically, we derive a formula for the effective capacity of a correlated Nakagami-m fading channel (note that Rayleigh fading is a special case of Nakagami-m fading with $m=1$ ). However, it is difficult to verify the accuracy of our derived EC formula via simulation since this requires that we should be able to compute the effective capacity via the closed-form formula, given the joint probability density function (pdf) of a correlated Nakagami-m fading channel, while at the same

\footnotetext{
${ }^{1}$ Marwan Krunz et al. [3], [4] used on-off processes (which model the codec-layer channel [11]) instead of Rayleigh/Rician/Nakagami-m fading with a practical Doppler spectrum (which model the radio-layer channel [11]).
} 
time, being able to simulate a channel gain process having the same joint pdf as that used in the closedform formula. To address this difficulty, we develop a methodology as below: we use an autoregressive (AR) model to simulate a correlated Nakagami-m fading channel; then use the corresponding correlation matrix of the AR model to obtain a Green matrix; then apply the Green matrix to the closed-form EC formula for calculating the EC. Simulation results show that our EC formula for a correlated Nakagami-m fading channel is accurate.

Furthermore, to facilitate the application of the EC theory to the design of practical QoS provisioning mechanisms in wireless networks, we propose a simple algorithm for estimating the EC of an arbitrary correlated Nakagami-m fading channel, given channel measurements. Simulation results demonstrate that our proposed EC estimation algorithm is accurate. Since our proposed EC estimation algorithm satisfies both accuracy and simplicity criteria, it shows the great suitability in practice.

The rest of the paper is organized as follows. Section II reviews the effective capacity theory. In Section III, we derive the EC formula for a special correlated Nakagami-m fading channel, for which the inverse of the correlation coefficient matrix is tridiagonal. Section IV presents a Green-matrix based method to obtain the EC formula for a general correlated Nakagami-m fading channel, given the correlation coefficient matrix of the channel. In Section V, we show the methodology for verifying the accuracy of our EC formula. Section VI presents an algorithm for estimating the effective capacity of an arbitrary correlated Nakagami-m fading channel, given channel gain measurements. In Section VII, we show the simulation results that verify the accuracy of the EC formula and the estimation algorithm. Section VIII concludes the paper.

\section{REVIEW OF THE EFFECTIVE CAPACITY}

The effective capacity [10] is a link-layer model in which a wireless link is modeled by two EC functions, namely the probability $\gamma(\mu)$ of nonempty buffer and the QoS exponent $\theta(\mu)$ of a connection. Both of them are functions of the source traffic rate $\mu$. The parsimonious EC channel model characterizes the capacity of a fading channel; mathematically, it is the dual of the effective bandwidth function [21], which characterizes the source randomness. Specifically, the key idea in the theory of effective capacity is that for a source that requires a communication delay bound $D_{\max }$ and can tolerate a delay-bound violation probability not more than $\varepsilon$, we need to limit its data rate, i.e., the maximum data rate is $\mu$, where $\mu$ is the solution to $\varepsilon=\gamma(\mu) e^{-\theta(\mu) D_{\max }}$, in which $\theta(\mu)=\mu \alpha^{-1}(\mu)$. Here $\alpha^{-1}(\cdot)$ is the inverse function of the effective capacity function $\alpha(\cdot)$. Next, we review the definition of the effective capacity.

Let $r(t)$ be the instantaneous channel capacity at time t. Define $S(t)=\int_{0}^{t} r(\tau) d \tau$, which is the service 
provided by the channel during the interval $[0, t]$. Suppose the stochastic process $r(t)$ is ergodic and stationary. Then the effective capacity function of $r(t)$ is defined by

$$
\alpha(u)=\frac{-\Lambda(-u)}{u}, \forall u>0
$$

where

$$
\Lambda(-u)=\lim _{t \rightarrow \infty} \frac{1}{t} \log E\left[e^{-u S(t)}\right]
$$

where $S(t)$ denotes the totally served traffic. Thus, if we know the effective capacity $\alpha(u)$, we can derive the QoS exponent function $\theta(\cdot)$ by $\theta(\mu)=\mu \alpha^{-1}(\mu)$; then, given the communication delay bound $D_{\max }$ and a delay-bound violation probability $\varepsilon$, we can estimate the probability $\gamma(\mu)$ of nonempty buffer through Monte Carlo simulation, given in Section V, and finally, constrain the source rate $\mu$ to satisfy the requested delay-bound violation probability, adapted to the channel condition. The effective capacity theory is very useful for the joint adaptive-modulation-and-coding, power control and resource allocation in practical communication systems such as wireless relay networks or MIMO systems.

\section{EfFective CApacity of a Special Correlated NAKAgAmi-M FAding Channel}

In this section, we derive the formula for the effective capacity of a special correlated Nakagami-m fading channel, for which the inverse of the correlation coefficient matrix is tridiagonal. This section is organized as below. Section III-A presents the joint pdf of Nakagami-m random vector that has a special correlation structure, i.e., the inverse of the correlation coefficient matrix being tridiagonal. In Section III$\mathrm{B}$, we derive the formula for the effective capacity of the special correlated Nakagami-m fading channel process.

\section{A. Joint pdf of a Nakagami-m Random Vector}

First, we consider a (scalar) Nakagami-m distributed random variable $X$; its pdf is given by

$$
f_{X}(x)=\frac{2 x^{2 m-1}}{\Gamma(m) \Omega^{m}} \exp \left(-\frac{x^{2}}{\Omega}\right), \quad x \geq 0, \quad m \geq \frac{1}{2}
$$

where $\Gamma(\cdot)$ is the Gamma function; $\Omega$ is a given parameter; $m$ is the degree of Nakagami-m distribution. Note that random variable $X$ here (and also in the rest of the paper) represents channel voltage gain instead of channel power gain, which is $X^{2}$.

There is a special property for a Nakagami-m distributed random variable with integer $m$. That is, a Nakagami-m distributed random variable with integer $m$, has the same pdf as that of the square root of the sum of squares of $m$ independent Rayleigh-distributed random variables or $2 m$ independent complex Gaussian random variables of zero-mean [22]. We can use this property to specify an N-dimensional 
Nakagami-m distributed random vector $\vec{X}$, by $N \times 2 m$ Gaussian random variables, which form an $N \times 2 m$ matrix. Denote $\left\{\mathbf{x}_{k},(k=1,2, \cdots, N)\right\}$ row vectors of the matrix; denote $\left\{\mathbf{y}_{k},(i=1,2, \cdots, 2 m)\right\}$ column vectors of the matrix. Assume that the column vectors $\left\{\mathbf{y}_{k},(k=1,2, \cdots, 2 m)\right\}$ are independent and identically distributed (i.i.d.). Assume that Gaussian random vector $\mathbf{y}_{k}$ has zero mean and correlation coefficient matrix $\Sigma$, which has dimension $N \times N$. The elements $\left\{\Sigma_{i, j},(i=1,2, \cdots, N ; j=1,2, \cdots, N)\right\}$ of $\Sigma$ are given as below: $\Sigma_{i, j}=1$ for $i=j$ and $\Sigma_{i, j}=\rho_{i, j}$ for $i \neq j$ with $0 \leq \rho_{i, j}<1$, where $\rho_{i, j}$ is the correlation coefficient between $i$-th element and $j$-th element of $\mathrm{N}$-dimensional random vector $\mathbf{y}_{k}$. $\Sigma=\left[\Sigma_{i, j}\right]$ is a tensor notation, which will be used in the rest of the paper.

It is known [23] that a general correlation coefficient matrix $\Sigma$ does not give a simple closed-form pdf for an N-dimensional Nakagami-m distributed random vector. However, if the inverse of the correlation coefficient matrix, $\Sigma^{-1}$, is tridiagonal, then the pdf for $\mathrm{N}$-dimensional Nakagami-m distributed random vector $\vec{X}$ is given by [23]

$$
f_{\vec{X}}\left(x_{1}, \cdots, x_{N}\right)=\frac{\left[\operatorname{det}\left(\Sigma^{-1}\right)\right]^{m} x_{1}^{m-1} x_{N}^{m} e^{-w_{N, N} x_{N}^{2} / 2}}{2^{m-1} \Gamma(m)} \prod_{k=1}^{N-1}\left[\left|w_{k, k+1}\right|^{-(m-1)} x_{k} e^{-w_{k, k} x_{k}^{2} / 2} I_{m-1}\left(\left|w_{k, k+1}\right| x_{k} x_{k+1}\right)\right]
$$

where $\operatorname{det}(\cdot)$ is the determinant of a matrix; $I_{\nu}(\cdot)$ is $\nu$-th order modified Bessel function of the first kind, i.e., $I_{m-1}(z)=\sum_{i=0}^{\infty} \frac{1}{i ! \Gamma(m+i)}\left(\frac{z}{2}\right)^{2 i+m-1}$, where $\sum_{i=0}^{\infty}=\lim _{n \rightarrow \infty} \sum_{i=0}^{n} ; w_{i, j}$ are elements of matrix $\Sigma^{-1}$, i.e., $\Sigma^{-1}=\left[w_{i, j}\right]$, using tensor notation; and in the above formula, $2 m$ has to be a positive integer which is also the case discussed in this paper. Hence, (4) is a special case of the pdf of a general Ndimensional Nakagami-m distributed random vector whose degree $m$ is allowed to be any positive real number satisfying $m \geq \frac{1}{2}$. In contrast, (4) is more general than the pdf of Chi-distribution since the degree $m$ of Chi-distribution has to be a positive integer while the degree $m$ in (4) is allowed to be half of any positive integer.

\section{B. Effective Capacity of a Special Correlated Nakagami-m Fading Channel}

Based on the pdf given in (4), we derive the EC formula for a special correlated Nakagami-m fading channel in this section.

Suppose that the wireless channel is a Nakagami-m flat fading channel in AWGN. Assume that we have perfect causal knowledge of the channel gains. We show how to calculate the effective capacity for this channel. Denote a sequence of $N_{t}$ measurements of the channel voltage gain over the duration $[0, t]$, spaced at a time-interval $\delta$ apart, by $\vec{x}=\left[x_{1}, x_{2}, \cdots, x_{N_{t}}\right]^{T}$, where $[\cdot]^{T}$ is a transpose operation. Without loss of generality, we have absorbed the constant noise variance into the definition of $x_{i}$, i.e., $x_{i}^{2}$ represents signal to noise ratio (SNR) under unity transmission power. The measurement $\left\{x_{i},\left(i=1,2, \cdots, N_{t}\right)\right\}$ is 
a realization of a random variable sequence denoted by $\left\{X_{i},\left(i=1,2, \cdots, N_{t}\right)\right\}$, which can be written as the vector $\vec{X}=\left[X_{1}, X_{2}, \cdots, X_{N_{t}}\right]^{T}$. The pdf of random vector $\vec{X}$ is given in the form of (4).

According to (1) and (2), to calculate the EC function, we need to know the expression of $E\left[e^{-u S(t)}\right]$; we derive it as below

$$
\begin{aligned}
& E\left[e^{-u S(t)}\right]=E\left[e^{-u \int_{0}^{t} r(\tau) d \tau}\right] \\
& \stackrel{(a)}{\approx} \int e^{-u\left(\sum_{i=1}^{N_{t}} \delta \times r\left(\tau_{i}\right)\right)} f\left(x_{1}, \cdots, x_{N_{t}}\right) d \vec{x} \\
& \stackrel{(b)}{=} \int e^{-u\left(\sum_{i=1}^{N_{t}} \delta \times \log \left(1+x_{i}^{2}\right)\right)} f\left(x_{1}, \cdots, x_{N_{t}}\right) d \vec{x} \\
& \stackrel{(c)}{\approx} \int_{0}^{\infty} e^{-u \delta\left(\sum_{k=1}^{N_{t}} x_{k}^{2}\right)} f\left(x_{1}, \cdots, x_{N_{t}}\right) d \vec{x} \\
& \stackrel{(d)}{=} \int_{0}^{\infty} e^{-u \delta\left(\sum_{k=1}^{N_{t}} x_{k}^{2}\right)} \frac{\left[\operatorname{det}\left(\Sigma^{-1}\right)\right]^{m} x_{1}^{m-1} x_{N_{t}}^{m} e^{-w_{N_{t}, N_{t}} x_{N_{t}}^{2} / 2}}{2^{m-1} \Gamma(m)} \prod_{k=1}^{N_{t}-1}\left[\left|w_{k, k+1}\right|^{-(m-1)} \cdot x_{k} \cdot e^{-w_{k, k} x_{k}^{2} / 2} .\right. \\
& \left.I_{m-1}\left(\left|w_{k, k+1}\right| x_{k} x_{k+1}\right)\right] d \vec{x} \\
& =\int \frac{\left[\operatorname{det}\left(\Sigma^{-1}\right)\right]^{m}}{2^{m-1} \Gamma(m)} x_{1}^{m-1} x_{N_{t}}^{m} e^{-\left(u \delta+w_{N_{t}, N_{t}} / 2\right) x_{N_{t}}^{2}} \prod_{k=1}^{N_{t}-1}\left[\left|w_{k, k+1}\right|^{-(m-1)} \cdot x_{k} \cdot e^{-\left(u \delta+w_{k, k} / 2\right) x_{k}^{2}} .\right. \\
& \left.\sum_{i=0}^{\infty} \frac{w_{k, k+1}^{m+2 i-1}\left(x_{k} x_{k+1}\right)^{m+2 i-1}}{2^{m-1+2 i} \Gamma(m+i) i !}\right] d \vec{x} \\
& =\int \frac{\left[\operatorname{det}\left(\Sigma^{-1}\right)\right]^{m}}{2^{m-1} \Gamma(m)} x_{1}^{m-1} x_{N_{t}}^{m} \prod_{j=1}^{N_{t}}\left[e^{-\left(u \delta+w_{j, j} / 2\right) x_{j}^{2}}\right] \prod_{k=1}^{N_{t}-1}\left[\sum_{i=0}^{\infty} \frac{w_{k, k+1}^{2 i} x_{k}^{m+2 i} x_{k+1}^{m+2 i-1}}{2^{m-1+2 i} \Gamma(m+i) i !}\right] d \vec{x} \\
& \stackrel{(e)}{=} \int A x_{1}^{m-1} x_{N_{t}}^{m} \prod_{j=1}^{N_{t}}\left[e^{-\left(u \delta+w_{j, j} / 2\right) x_{j}^{2}}\right] \prod_{k=1}^{N_{t}-1}\left[\sum_{i=0}^{\infty} \frac{w_{k, k+1}^{2 i} x_{k}^{m+2 i} x_{k+1}^{m+2 i-1}}{B_{i}}\right] d \vec{x} \\
& \stackrel{(f)}{=} \int A x_{1}^{m-1} x_{N_{t}}^{m} \prod_{j=1}^{N_{t}}\left[e^{-\left(u \delta+w_{j, j} / 2\right) x_{j}^{2}}\right] \times \\
& \sum_{i_{1}=0}^{\infty} \sum_{i_{2}=0}^{\infty} \cdots \sum_{i_{N_{t}-1}=0}^{\infty} C\left(i_{1}, \cdots, i_{N_{t}-1}\right) x_{1}^{m+2 i_{1}} x_{2}^{2 m+2 i_{1}+2 i_{2}-1} \cdots x_{N_{t}-1}^{2 m+2 i_{N_{t}-2}+2 i_{N_{t}-1}-1} x_{N_{t}}^{2 m+2 i_{N_{t}-1}-1} d \vec{x} \\
& \stackrel{(g)}{=} \sum_{i_{1}=0}^{\infty} \sum_{i_{2}=0}^{\infty} \cdots \sum_{i_{N_{t}-1}=0}^{\infty} \frac{A}{2^{N_{t}}} C\left(i_{1}, \cdots, i_{N_{t}-1}\right) \prod_{j=1}^{N_{t}}\left[\left(u \delta+w_{j, j} / 2\right)^{-\left(m+i_{j-1}+i_{j}\right)} \Gamma\left(m+i_{j-1}+i_{j}\right)\right]
\end{aligned}
$$

where (a) is due to approximation of integration (in the exponent) by summation over equi-spacing samples $\tau_{i}$; (b) $r\left(\tau_{i}\right)=\log \left(1+x_{i}^{2}\right)$ since $r\left(\tau_{i}\right)$ is the instantaneous AWGN channel capacity at time $\tau_{i}$ and $x_{i}^{2}$ is SNR under the assumption that the transmission power and noise variance are absorbed into $x_{i}^{2}$; (c) due to $\log \left(1+|x|^{2}\right) \approx|x|^{2}$; (d) due to substituting $f\left(x_{1}, \cdots, x_{N_{t}}\right)$ by (4); (e) due to the substitutions $A=\frac{\left[\operatorname{det}\left(\Sigma^{-1}\right)\right]^{m}}{2^{m-1} \Gamma(m)}$ and $B_{i}=2^{m-1+2 i} \Gamma(m+i) i$; ; (f) due to the substitution $C\left(i_{1}, \cdots, i_{N_{t}-1}\right)=\prod_{k=1}^{N_{t}-1} \frac{w_{k, k+1}^{2 i_{k}}}{B_{i_{k}}}$; and (g) due to $\Gamma(x)=\int t^{x-1} e^{-t} d t$. Moreover, $i_{0}=i_{N_{t}}=0$ holds in the derivation. Since we use the approximation $\log \left(1+|x|^{2}\right) \approx|x|^{2}$, (5) is accurate for relatively low SNR. Moreover, by Monte Carlo 
simulations, the speed of the error growth between the analytical and simulated results goes faster as the SNR increases when it falls into the region SNR $>17 \mathrm{~dB}$, i.e., the corresponding error larger than $10 \%$. In particular, a practical 3G WCDMA system works under relatively low SNR region (about 5dB); hence, our approximation of a low SNR case has its practical use.

From (1) and (2), we have

$$
\begin{aligned}
\alpha(u) & =\lim _{t \rightarrow \infty} \frac{-1}{u t} \log E\left[e^{-u S(t)}\right] \\
& \stackrel{(a)}{\approx} \frac{-1}{u N_{t} \delta} \log E\left[e^{-u \widetilde{S}(t)}\right] \\
\stackrel{(b)}{=} & \frac{-1}{u N_{t} \delta} \log \left(\sum_{i_{1}=0}^{\infty} \sum_{i_{2}=0}^{\infty} \cdots \sum_{i_{N_{t}-1}=0}^{\infty} \frac{A}{2^{N_{t}}} C\left(i_{1}, \cdots, i_{N_{t}-1}\right) \prod_{j=1}^{N_{t}}\left[\left(u \delta+\frac{w_{j, j}}{2}\right)^{-\left(m+i_{j-1}+i_{j}\right)} \Gamma\left(m+i_{j-1}+i_{j}\right)\right]\right) \\
\stackrel{(c)}{=} & \frac{-1}{u N_{t} \delta} \log \left(\sum_{i_{1}=0}^{\mathbb{K}} \sum_{i_{2}=0}^{\mathbb{K}} \cdots \sum_{i_{N_{t}-1}=0}^{\mathbb{K}} \frac{A}{2^{N_{t}}} C\left(i_{1}, \cdots, i_{N_{t}-1}\right) \prod_{j=1}^{N_{t}}\left[\left(u \delta+\frac{w_{j, j}}{2}\right)^{-\left(m+i_{j-1}+i_{j}\right)} \Gamma\left(m+i_{j-1}+i_{j}\right)\right]\right) \\
& +o\left(2^{-N_{t}(\mathbb{K}+1)}\right)
\end{aligned}
$$

where (a) is due to $t=\left(N_{t}-1\right) \delta \approx N_{t} \delta, \widetilde{S}(t)=\sum_{i=1}^{N_{t}} \delta \times r\left(\tau_{i}\right)$, and we assume that $N_{t}$ is sufficiently large; (b) is due to (5); and (c) we partition the sum of infinite number of terms into the sum of finite number of terms plus a remainder term $o\left(2^{-N_{t}(\mathbb{K}+1)}\right)$, similar to the $\mathbb{K}$-th order Taylor approximation of a differentiable function.

Since $\alpha(u)$ is defined for a continuous time system, we now define the effective capacity for a discrete time system, denoted by $\alpha^{(d)}(u)$, which is based on (6), i.e., for some integer $\mathbb{K} \geq 0$,

$\alpha^{(d)}(u)=\frac{-1}{u N_{t} \delta} \log \left(\sum_{i_{1}=0}^{\mathbb{K}} \sum_{i_{2}=0}^{\mathbb{K}} \cdots \sum_{i_{N_{t}-1}=0}^{\mathbb{K}} \frac{A}{2^{N_{t}}} C\left(i_{1}, \cdots, i_{N_{t}-1}\right) \prod_{j=1}^{N_{t}}\left[\left(u \delta+\frac{w_{j, j}}{2}\right)^{-\left(m+i_{j-1}+i_{j}\right)} \Gamma\left(m+i_{j-1}+i_{j}\right)\right]\right)$

The error $\left|\alpha(u)-\alpha^{(d)}(u)\right|$ is very small for sufficiently large $N_{t}$ because the remainder term $o\left(2^{-N_{t}(\mathbb{K}+1)}\right)$ decreases exponentially with the increase of $N_{t}$. Hence, $\mathbb{K}$ can be small as long as $N_{t}$ is sufficiently large. Actually, $\mathbb{K}=0$ is enough to guarantee accuracy because the product of $N_{t}$ terms indexed by $i_{j}$, i.e., $\prod_{j=1}^{N_{t}} \frac{\Gamma\left(m+i_{j-1}+i_{j}\right) \Gamma\left(m+i_{j}+i_{j+1}\right)}{2^{2 i_{j}} \cdot i_{j} ! \cdot \Gamma\left(m+i_{j}\right)}$ in (7), approaches 0 when $N_{t}$ is sufficiently large and this is verified by our numerical results. As shown in Fig. 3 and Fig. $4, \mathbb{K}=0$ can produce relatively accurate results. More importantly, when we choose $\mathbb{K}=0$, the computation complexity of (7) increases linearly with $N_{t}$ rather than exponential growth. Hence, we have

$$
\alpha^{(d)}(u)=\frac{-1}{u N_{t} \delta} \log \left(\frac{A}{2^{N_{t}}} C\left(i_{1}=0, \cdots, i_{N_{t}-1}=0\right) \prod_{j=1}^{N_{t}}\left[\left(u \delta+\frac{w_{j, j}}{2}\right)^{-m} \Gamma(m)\right]\right)
$$

The simple EC formula that we obtain in (8) has significant implication. Its simplicity allows the use of the effective capacity in the design of practical QoS provisioning mechanisms. 
Note that the EC formula (8) is only valid for a special correlated Nakagami-m fading channel, for which the inverse of the correlation coefficient matrix, i.e., $\Sigma^{-1}$, is tridiagonal. Next, we show how to compute the effective capacity of a general correlated Nakagami-m fading channel.

\section{Effective Capacity of A general CorRelated NaKagami-M Fading Channel}

The EC formula (8) derived in Section III-B cannot be directly used to compute the effective capacity of a general correlated Nakagami-m fading channel, for which the inverse of the correlation coefficient matrix, i.e., $\Sigma^{-1}$, is not tridiagonal. To address this, we choose the mathematical tool based on Green's matrix given in [23] whose validity has been verified. The reason why we use a Green's matrix based approach is two-folded. First, the inverse of a Green's matrix is tridiagonal, making it possible to use (8) for calculating the effective capacity of the channel. Specifically, according to a theorem in [24], a symmetric irreducible matrix is tridiagonal if and only if its inverse is a Green's matrix.

Our Green's matrix based approach is as follows. Given an arbitrary correlation coefficient matrix $\Sigma$, whose inverse is not tridiagonal, we project the matrix $\Sigma$ onto the space of Green's matrices; denote its projection by $G^{*}$. Then we compute the effective capacity via (8), under the assumption that the correlation coefficient matrix of the Nakagami-m fading channel is $G^{*}$.

Now, we show how to project an arbitrary correlation coefficient matrix $\Sigma$ of dimension $N_{t} \times N_{t}$ onto the space of Green's matrices of dimension $N_{t} \times N_{t}$ [23]. We first review the Green's matrix [24], [25]. Similar to the correlation coefficient matrix $\Sigma$, a Green's matrix, $G$, is also symmetric. A Green's matrix satisfies the following constraint

$$
G=\left[G_{i, j}\right]=\left[\begin{array}{cccc}
u_{1} v_{1} & u_{1} v_{2} & \cdots & u_{1} v_{N_{t}} \\
u_{1} v_{2} & u_{2} v_{2} & \cdots & u_{2} v_{N_{t}} \\
\vdots & & \ddots & \vdots \\
u_{1} v_{N_{t}} & u_{2} v_{N_{t}} & \cdots & u_{N_{t}} v_{N_{t}}
\end{array}\right]
$$

where $u_{i}\left(i=1, \ldots, N_{t}\right)$ and $v_{j}\left(j=1, \ldots, N_{t}\right)$ are real numbers; and $G^{-1}$ is tridiagonal. Given the correlation coefficient matrix $\Sigma$ of a general correlated Nakagami-m fading channel, we can find its Green's matrix projection $G^{*}$ by solving the following least squares problem,

$$
\begin{gathered}
\min _{G,\left\{u_{i}\right\},\left\{v_{j}\right\}} \sum_{i} \sum_{j}\left(G_{i, j}-\Sigma_{i, j}\right)^{2} \\
\text { s.t. } \quad G \text { satisfies }(9), \\
u_{i} v_{i}=1, \quad \forall i .
\end{gathered}
$$

Constraint (10c) is due to the fact that $\Sigma_{i, i}=1(\forall i)$ for a general correlated Nakagami-m fading channel. Moreover, ref. [23] has verified the accuracy of replacing a correlation coefficient matrix $\Sigma$ by its projected Green's matrix, in modeling a general correlated Nakagami-m fading channel. 
To solve (10) for $G^{*}$, we can use the Levenberg-Marquardt Algorithm (LMA) [26], which was designed to solve nonlinear curve-fitting problems under the least-squares criterion. The LMA interpolates between the Gauss-Newton algorithm (GNA) and the method of gradient descent and LMA is more robust than the GNA, which means that in many cases it finds a solution even if it starts very far off the final minimum so LMA could also be viewed as GNA improved with trust region approach. Moreover, it is very convenient for us to directly use it by the matlab function, such as "Isqcurvefit(.)", etc.

Algorithm 1 summarizes our method to compute the effective capacity of a general correlated Nakagami$\mathrm{m}$ fading channel.

Algorithm 1: Computing the effective capacity of a general correlated Nakagami-m fading channel.

Input: Correlation coefficient matrix $\Sigma$ of a correlated Nakagami-m fading channel.

If $\Sigma$ is a Green matrix

Compute the effective capacity of the channel $\alpha^{(d)}(u)$ via (8);

Else

Solve (10) for $G^{*}$ by the Levenberg-Marquardt algorithm;

Compute $\alpha^{(d)}(u)$ via (8), using $G^{*}$ as the correlation coefficient matrix of the channel; Endif

Output: $\alpha^{(d)}(u)$.

The computational complexity of Algorithm 1 is given as below. The computational complexity of the step in the first branch, i.e., computing the EC of the channel $\alpha^{(d)}(u)$ via (8), is $\mathcal{O}\left(N_{t}\right)$, which means it has linear complexity, since we take the first $K$ items as an approximation in Step (c) of Eqn. (6). In contrast, the original formula in Step (b) of Eqn. (6) has an exponential computational complexity $\mathcal{O}\left(2^{\left.N_{t}\right)}\right.$, making its computation unpractical. In addition, for the first step in the second branch, i.e., solving (10) for $G^{*}$ by the Levenberg-Marquardt Algorithm, its computational complexity determined by the dimension of the correlated matrix is given by $\mathcal{O}\left(N_{t}^{2}\right)$ [26], which means it can converge quickly. Then, the total computational complexity of the two steps in this branch is on the order of $\mathcal{O}\left(N_{t}^{2}\right)$. Thus, Algorithm 1 has reasonable complexity so that it can be implemented in practical systems.

\section{Methodology for Verifying the Accuracy of Our EC formula}

In the previous section, we proposed Algorithm 1 to compute the effective capacity of a general correlated Nakagami-m fading channel. However, it is difficult to verify the accuracy of Algorithm 1 and the EC formula (8) via simulation since this requires that we should be able to compute the effective 
capacity via the closed-form formula, given the joint pdf of a correlated Nakagami-m fading channel, while at the same time, being able to simulate a channel gain process having the same joint pdf as that used in the closed-form formula. To address this difficulty, in this section, we present a methodology to verify the accuracy of Algorithm 1 and the EC formula (8).

The key idea of our methodology is as below: we use an AR(1) model to simulate a correlated Nakagami$\mathrm{m}$ fading channel. (This is a widely used model to generate an exponentially correlated fading channel); then use the corresponding correlation matrix of the $\operatorname{AR}(1)$ model to obtain a Green matrix, which approximates the correlation matrix of the AR(1) model; then apply the Green matrix to the closed-form EC formula (8) for calculating the EC. Specifically, our verification methodology is given as below.

1) Simulate a discrete-time Nakagami-m fading channel with an $\operatorname{AR}(1)$ model where $\operatorname{AR}(n)$ is an autoregressive model of order $n$; hence the Nakagami-m fading channel has exponential correlation. The details of how to simulate this channel is given in Section VII-B.1. The correlation coefficient matrix $\Sigma$ of the exponentially correlated Nakagami-m fading channel can be analytically derived and is given in (29) in Section VII-B.1.

2) Given $\Sigma$ in (29), run Algorithm 1 and obtain $\alpha^{(d)}(u)$.

3) Simulate the communication system shown in Fig. 2, where the source rate $\mu$ is constant.

4) Collect the following measurements from the queueing system at the $n$-th sampling epoch ( $n=$ $\left.1,2, \cdots, N_{s}\right): S(n)$ the indicator of whether a packet is in service $(S(n) \in\{0,1\}), Q(n)$ the number of bits in the queue (excluding the packet in service), and $\tau(n)$ the remaining service time of the packet in service (if there is one in service).

5) Calculate the measured EC function $\alpha_{s}(u)$ by the following procedure.

$$
\begin{aligned}
\hat{\gamma} & =\frac{1}{N_{s}} \sum_{t=1}^{N_{s}} S(n), \\
\hat{q} & =\frac{1}{N_{s}} \sum_{t=1}^{N_{s}} Q(n), \\
\hat{\tau_{s}} & =\frac{1}{N_{s}} \sum_{t=1}^{N_{s}} \tau(n), \\
\hat{\theta} & =\frac{\hat{\gamma} \times \mu}{\mu \times \hat{\tau}_{s}+\hat{q}}, \\
\alpha_{s}(u) & =\mu, \quad \text { for } u=\hat{\theta} / \mu .
\end{aligned}
$$


6) Verify whether the error $\left|\alpha^{(d)}(u)-\alpha_{s}(u)\right|$ is small.

We follow the above verification methodology and conduct simulations in Section VII-B.1. As shown in Fig. 3 and Fig. 4, the curve for $\alpha^{(d)}(u)$ (obtained from analysis) matches that for $\alpha_{s}(u)$ (obtained from the simulation). This verifies that Algorithm 1 and the EC formula (8) are accurate.

Actually, for an arbitrary correlated Nakagami-m fading channel, the accuracy of Algorithm 1 and the EC formula (8) is also indirectly verified through the simulation results based on the estimation algorithm introduced in Section VI, as shown in Figs. 7 and 8.

\section{Algorithm For estimating THE EFFECTIVE CAPACITY OF AN ARBitrary CORRELATED NAKAGAMI-M CHANNEL}

Now, we know that Algorithm 1 is accurate. The next question is how to use Algorithm 1 in practice.

In a practical system, we cannot directly use Algorithm 1 to compute the effective capacity since the correlation coefficient matrix $\Sigma$ of a Nakagami-m fading channel is not known a priori; in other words, we need to estimate $\Sigma$ from the channel gain measurements in order to use Algorithm 1. Next, we first present Proposition 1 that quantifies the relationship between $\Sigma$ and the statistics of channel gains; then, we propose an algorithm to estimate $\Sigma$ and the effective capacity of the channel, given channel gain measurements.

Proposition 1: Assume N-dimensional Nakagami-m distributed random vector $\left[X_{1}, \cdots, X_{N}\right]^{T}$ is specified by

$$
\left\{\begin{array}{c}
X_{1}^{2}=Y_{1,1}^{2}+Y_{1,2}^{2}+\cdots+Y_{1,2 m}^{2} \\
X_{2}^{2}=Y_{2,1}^{2}+Y_{2,2}^{2}+\cdots+Y_{2,2 m}^{2} \\
\cdots \\
X_{N}^{2}=Y_{N, 1}^{2}+Y_{N, 2}^{2}+\cdots+Y_{N, 2 m}^{2}
\end{array}\right.
$$

where $Y_{k, l}(k=1, \cdots, N$ and $l=1, \cdots, 2 m)$ are identically distributed Gaussian random variables with zero mean and variance $\sigma^{2}$; for any $k(k=1, \cdots, N), Y_{k, 1}, Y_{k, 2}, \cdots, Y_{k, 2 m}$ are independent; the column vectors $\left\{\left[Y_{1, l}, Y_{2, l}, \cdots, Y_{N, l}\right]^{T},(l=1, \cdots, 2 m)\right\}$ are i.i.d. with zero mean and correlation coefficient matrix $\Sigma=\left[\Sigma_{i, j}\right]$, which has dimension $N \times N$, and $\Sigma_{i, j}=E\left[Y_{i, l} Y_{j, l}\right] / \sigma^{2}$. Then, the following holds.

1) $X_{1}, \cdots, X_{N}$ are identically distributed random variables.

2) Denote $\mu_{X}$ the mean of $X_{i}(i=1, \cdots, N)$; and denote $\nu_{X}^{2}$ the variance of $X_{i}(i=1, \cdots, N)$. Then

$$
\operatorname{Cov}\left(X_{i}^{2}, X_{j}^{2}\right)=R\left(X_{i}^{2}, X_{j}^{2}\right)-\left(\mu_{X}^{2}+\nu_{X}^{2}\right)^{2}
$$

where $\operatorname{Cov}(\cdot, \cdot)$ and $R(\cdot, \cdot)$ denote the covariance and correlation between two random variables, respectively. 
3) Correlation coefficient matrix $\Sigma=\left[\Sigma_{i, j}\right]$ is given by

For the proof of Proposition 1, see Appendix A.

$$
\Sigma_{i, j}=\frac{\sqrt{m \times \operatorname{Cov}\left(X_{i}^{2}, X_{j}^{2}\right)}}{\mu_{X}^{2}+\nu_{X}^{2}}
$$

Proposition 1 quantifies the relationship between $\Sigma$ and the statistics of channel gains $\left\{X_{i},(i=\right.$ $1,2, \cdots, N)\}$. Next, we present an algorithm to estimate $\Sigma$ and the effective capacity of the channel, given channel gain measurements.

Algorithm 2: Estimating $\Sigma$ of dimension $N_{t} \times N_{t}$ and the effective capacity:

1) Obtain $N_{e}$ measurements of the channel gain; denote these measurements by $\mathbf{x}=\left[x(1), x(2), \cdots, x\left(N_{e}\right)\right]^{T}$, where $x(k)\left(k=1, \cdots, N_{e}\right)$ are real-valued channel voltage gains.

2) Given channel gain measurements $\mathbf{x}$, estimate $\mu_{X}, \nu_{X}^{2}$, and $R\left(X_{i}^{2}, X_{j}^{2}\right)$ via the following equations:

$$
\begin{gathered}
\widehat{\mu}_{X}=\frac{1}{N_{e}} \sum_{k=1}^{N_{e}} x(k) \\
\widehat{\nu}_{X}^{2}=\frac{1}{N_{e}-1} \sum_{k=1}^{N_{e}}\left(x(k)-\widehat{\mu}_{X}\right)^{2} \\
\widehat{R}\left(X_{i}^{2}, X_{j}^{2}\right)=\frac{1}{N_{e}-(j-i)} \sum_{k=1}^{N_{e}-(j-i)} x^{2}(k) x^{2}(k+j-i), \quad i=1, \cdots, N_{t}-1, \quad j=i+1, \cdots, N_{t} \\
\widehat{R}\left(X_{j}^{2}, X_{i}^{2}\right)=\widehat{R}\left(X_{i}^{2}, X_{j}^{2}\right), \quad i=1, \cdots, N_{t}-1, \quad j=i+1, \cdots, N_{t} \\
\widehat{R}\left(X_{i}^{2}, X_{i}^{2}\right)=\frac{1}{N_{e}} \sum_{k=1}^{N_{e}} x^{4}(k), \quad i=1, \cdots, N_{t}
\end{gathered}
$$

3) Estimate the covariance $\operatorname{Cov}\left(X_{i}^{2}, X_{j}^{2}\right)$ via (17), i.e.,

$$
\widehat{\operatorname{Cov}}\left(X_{i}^{2}, X_{j}^{2}\right)=\widehat{R}\left(X_{i}^{2}, X_{j}^{2}\right)-\left(\widehat{\mu}_{X}^{2}+\widehat{\nu}_{X}^{2}\right)^{2}, \quad i=1, \cdots, N_{t}, \quad j=1, \cdots, N_{t}
$$

4) Estimate the correlation coefficient matrix $\Sigma=\left[\Sigma_{i, j}\right]$ via (18), i.e.,

$$
\widehat{\Sigma}_{i, j}=\frac{\sqrt{m \times \widehat{\operatorname{Cov}}\left(X_{i}^{2}, X_{j}^{2}\right)}}{\widehat{\mu}_{X}^{2}+\widehat{\nu}_{X}^{2}}
$$

5) Give $\left[\widehat{\Sigma}_{i, j}\right]$, compute the estimate of the effective capacity, denoted by $\widehat{\alpha}_{e}(u)$, via Algorithm 1. 
In Algorithm 2, we assume the channel gain process is ergodic and stationary so that the estimators in (19) through (25) are consistent ${ }^{2}$. In addition, (20) is an unbiased estimate of $\nu_{X}^{2}$.

We implement Algorithm 2 and conduct simulations in Section VII-B.3. As shown in Figs. 7 and 8, the curve for $\widehat{\alpha}_{e}(u)$ (obtained via Algorithm 2) agrees well with that for $\alpha_{s}(u)$ (obtained via (11) through (15) from the simulation). This shows that Algorithm 2 provides an accurate estimate of the effective capacity of an arbitrary correlated Nakagami-m fading channel.

\section{Simulation Results}

\section{A. Simulation Setting}

We simulate the discrete-time system depicted in Fig. 2. In this system, the data source generates packets at a constant rate $\mu$. Generated packets are first sent to the (infinite) buffer at the transmitter, whose queue length is $Q(n)$, where $n$ refers to the $n$-th sample-interval. The head-of-line packet in the queue is transmitted over the fading channel at data rate $r(n)$. The fading channel has a random voltage gain $X(n)$; and $X^{2}(n)$ is channel power gain. We use a fluid model, that is, the size of a packet is infinitesimal. In practical systems, the results presented here will have to be modified to account for finite packet sizes.

We assume that the transmitter has perfect knowledge of the current channel gains $X(n)$ at each sampleinterval. Therefore, it can use rate-adaptive transmissions and ideal channel codes, to transmit packets without decoding errors. Thus, the transmission rate $r(n)$ is equal to the instantaneous (time-varying) capacity of the fading channel, as below,

$$
r(n)=B_{c} \log _{2}\left(1+X^{2}(n) \times P_{0} / \sigma_{n}^{2}\right)
$$

where $B_{c}$ denotes the channel bandwidth, and the transmission power $P_{0}$ and noise variance $\sigma_{n}^{2}$ are assumed to be constant. The average SNR is fixed in each simulation run and the average SNR $S N R_{a v g}=$ $E\left[X^{2}(n) \times P_{0} / \sigma^{2}\right]$. Since we set $E\left[X^{2}(n)\right]=1$, we have $S N R_{\text {avg }}=E\left[X^{2}(n) \times P_{0} / \sigma^{2}\right]=P_{0} / \sigma^{2}$.

In our simulations, the sampling interval $\delta$ is set to 1 milli-second. This is not too far from reality, since 3G WCDMA systems already incorporate rate adaptation on the order of 10 milli-second [27]. Meanwhile, for the current enhanced HSPA systems, they have much faster adaption speed, i.e., on the order of 2 milli-second. Each simulation run is 10,000-second long for all the scenarios, in order to obtain good estimate by the Monte Carlo method. Since the sampling interval is 1 milli-second, we have 10,000,000 samples for estimation; i.e., $N_{s}=10^{7}$.

Table I lists the parameters used in our simulations.

\footnotetext{
${ }^{2} \mathrm{~A}$ consistent sequence of estimators is a sequence of estimators that converge in probability to the quantity being estimated as the index (usually the sample size) grows without bound.
} 
TABLE I

SIMULATION PARAMETERS

\begin{tabular}{|c|c|}
\hline Maximum Doppler frequency $f_{m}$ & $15 / 100 \mathrm{~Hz}$ \\
Channel bandwidth $B_{c}$ & $100 \mathrm{kHz}$ \\
Average SNR & $0 \mathrm{~dB}$ to $10 \mathrm{~dB}$ \\
Sampling-interval $\delta$ & $1 \mathrm{~ms}$ \\
Number of samples $N_{t}$ & 1024 \\
Number of samples $N_{s}$ & $10^{7}$ \\
Number of samples $N_{e}$ & $10^{7}$ \\
\hline
\end{tabular}

\section{B. Simulation Results}

1) Accuracy of Algorithm 1 and the EC formula (8): In this section, we verify the accuracy of Algorithm 1 and the EC formula (8) through simulations.

As mentioned in Section V, we will simulate an exponentially correlated Nakagami-m fading channel so that we can compute the effective capacity via the closed-form formula, given the joint pdf of the fading channel, while at the same time, being able to simulate a channel gain process having the same joint pdf as that used in the closed-form formula.

Next we show how to simulate $N_{s}$ samples of an exponentially-correlated Nakagami-m fading channel. We make the same assumption as that in Proposition 1. We want to generate $N_{s}$ samples of channel voltage gains. This is equivalent to generating an $N_{s}$-dimensional Nakagami-m distributed random vector $\left[X_{1}, \cdots, X_{N_{s}}\right]^{T}$, which can be specified by

$$
X_{k}^{2}=Y_{k, 1}^{2}+Y_{k, 2}^{2}+\cdots+Y_{k, 2 m}^{2}, \quad \text { for } k=1, \cdots, N_{s}
$$

where $Y_{k, l}\left(k=1, \cdots, N_{s}\right.$ and $\left.l=1, \cdots, 2 m\right)$ are identically distributed Gaussian random variables with zero mean and unit variance; for any $k\left(k=1, \cdots, N_{s}\right), Y_{k, 1}, Y_{k, 2}, \cdots, Y_{k, 2 m}$ are independent; the column vectors $\left\{\left[Y_{1, l}, Y_{2, l}, \cdots, Y_{N_{s}, l}\right]^{T},(l=1, \cdots, 2 m)\right\}$ are i.i.d. with zero mean and correlation coefficient matrix $\Sigma=\left[\Sigma_{i, j}\right]$, which has dimension $N_{s} \times N_{s}$, and $\Sigma_{i, j}=E\left[Y_{i, l} Y_{j, l}\right]$.

Now, we describe how to generate $\left[X_{1}, \cdots, X_{N_{s}}\right]^{T}$ in detail. We first generate $2 m$ i.i.d. Gaussian random variables with zero mean and unit variance and denote them by $Y_{1,1}, Y_{1,2}, \cdots, Y_{1,2 m}$; then use (27) with $k=1$ to generate $X_{1}$. Then we use the following $\operatorname{AR}(1)$ model to recursively generate $Y_{k, l}\left(k=2, \cdots, N_{s}\right.$ and $l=1, \cdots, 2 m)$ :

$$
Y_{k, l}=\beta Y_{k-1, l}+\sqrt{1-\beta^{2}} g_{k-1, l}, \quad \text { for } k=2, \cdots, N_{s} \text { and } l=1, \cdots, 2 m
$$

where $\left\{g_{k, l}\left(k=1, \cdots, N_{s}-1 ; l=1, \cdots, 2 m\right)\right\}$ are i.i.d. Gaussian random variables with zero mean and unit variance; $\beta$ is a real number satisfying $0<\beta<1$. Specifically, for a given $k(k>1)$, we first generate i.i.d. Gaussian random variables $\left\{g_{k, l}(l=1, \cdots, 2 m)\right\}$; then use $(28)$ to generate $\left\{Y_{k, l}(l=1, \cdots, 2 m)\right\}$; 
then use (27) to generate $X_{k}$; then increase $k$ by one and repeat this process. By doing so, we obtain $X_{1}, \cdots, X_{N_{s}}$. Since we recursively use AR(1) model to generate the channel gain $X_{k}$, the total number of samples $N_{s}$ can be arbitrarily large, which is an advantage of our simulation method.

From (28), we can analytically derive the correlation coefficient matrix $\Sigma$ of dimension $N_{s} \times N_{s}$, which is given as below

$$
\Sigma=\left[\begin{array}{ccccccc}
1 & \beta & \beta^{2} & \beta^{3} & \ldots & \beta^{N_{s}-2} & \beta^{N_{s}-1} \\
\beta & 1 & \beta & \beta^{2} & \cdots & \beta^{N_{s}-3} & \beta^{N_{s}-2} \\
\vdots & \vdots & \vdots & \vdots & \vdots & \vdots & \vdots \\
\beta^{N_{s}-1} & \beta^{N_{s}-2} & \cdots & \cdots & \cdots & \beta & 1
\end{array}\right]
$$

The dimension $N_{s} \times N_{s}$ of $\Sigma$ in (29) is too large, which leads to very high complexity in computing the effective capacity $\alpha^{(d)}(u)$ via (8). To reduce the computational complexity of (8), we need to approximate $\Sigma$ in (29) by a matrix of a lower dimension. As we know, $0<\beta<1$ and hence $\beta^{m}$ is very small for a large $m$, i.e., $Y_{k, l}$ and $Y_{k+m, l}(\forall l)$ can be considered uncorrelated for a large $m$. We choose $\Sigma$ of dimension $N_{t} \times N_{t}$ (where $N_{t}=1024$ ) to approximate $\Sigma$ of dimension $N_{s} \times N_{s}$ (where $N_{s}=10^{7}$ ), since $\beta^{N_{t}-1}$ is extremely small. This guarantees that the effective capacity obtained by $\Sigma$ of dimension $N_{t} \times N_{t}$ via (8) is almost the same as that obtained by $\Sigma$ of dimension $N_{s} \times N_{s}$. For $\Sigma$ of dimension $N_{t} \times N_{t}$, we can further simplify it by replacing an element $\beta^{m}$ by 0 if $\beta^{m}<\Gamma_{t h}$, where $\Gamma_{t h}>0$ is a preset small value.

Next, we give an example. Assume $\beta=0.3679$ and $\Gamma_{t h}=0.02$. Then $m=4$ is the least positive integer satisfying $\beta^{m}<0.02$. Hence, we replace all the elements $\beta^{m}$ ( $\left.m \geq 4\right)$ in (29) by zero. Denote the resulting matrix of dimension $N_{t} \times N_{t}$ by $\widetilde{\Sigma}$. Using $\widetilde{\Sigma}$ as the input, we run Algorithm 1 to obtain the effective capacity $\alpha^{(d)}(u)$. To obtain the measured EC function $\alpha_{s}(u)$, we collect measurements from the simulated queueing system shown in Fig. 2, and calculate $\alpha_{s}(u)$ via (11) through (15).

Figs. 3 and 4 show the measured EC function $\alpha_{s}(u)$ obtained from the simulation and the EC function $\alpha^{(d)}(u)$ obtained from the analysis, under different Doppler frequencies and different values of $m$. In the simulation and the analysis, the average SNR is $10 \mathrm{~dB}$. It is observed that the curve for $\alpha^{(d)}(u)$ gives good agreement with that for $\alpha_{s}(u)$. The slight difference between $\alpha^{(d)}(u)$ and $\alpha_{s}(u)$ is mainly caused by two approximations in our analysis, i.e., 1) $\log \left(1+|x|^{2}\right) \approx|x|^{2}$, and 2) considering only finite number of terms $(\mathbb{K}=0)$ in $(7)$ instead of infinite number of terms $(\mathbb{K}=\infty)$. The results in Figs. 3 and 4 verify that Algorithm 1 and the EC formula (8) are accurate.

Meanwhile, we also compare our analytical EC function $\alpha^{(d)}(u)$ with the measured EC function $\alpha_{s}^{Z}(u)$ from the simulation by using Zhang's direct-sum decomposition principle and statistically parameters mapping model [28] for an arbitrary correlated Nakagami-m fading channel. It is observed that the two results agree with each other in terms of shape and trend. We also observe that the result obtained under 
Zhang's model is less accurate than that obtained under the AR(1) model. This may be due to less accurate estimation of parameter $\beta$ in the rigidly exponential correlation matrix.

2) Comparing our EC Formula with Wu and Negi's Formula for Rayleigh Fading: Previously, an EC formula [10] was derived for a correlated Rayleigh fading channel, which is a special Nakagami-m fading channel with $m=1$. Their EC formula is given as below

$$
\alpha(u)=\frac{\int \log (u S(f)+1) d f}{u}, \quad u>0,
$$

where $S(f)$ is the power spectral density of the Rayleigh fading channel.

We use the same simulation setting as that in Section VII-B.1 with $m=1$. The average SNR is $5 \mathrm{~dB}$. Figs. 5 and 6 plot 1) the measured EC function $\alpha_{s}(u)$ obtained from the simulation, 2) the EC function $\alpha^{(d)}(u)$ obtained from the analysis of this paper with $m=1$, and 3) the EC function $\alpha(u)$ obtained from (30), under different Doppler frequencies. From the figures, it is observed that $\alpha^{(d)}(u)$ and $\alpha(u)$ obtained by two different EC formulae are consistent, and both of them give good agreement with the measured EC function $\alpha_{s}(u)$. The small difference between $\alpha^{(d)}(u)$ and $\alpha(u)$ is mainly caused by the approximation due to consideration of only finite number of terms $(\mathbb{K}=0)$ in $(7)$ instead of infinite number of terms $(\mathbb{K}=\infty)$. Hence, the results here demonstrate that the EC formula in this paper is consistent with the EC formula, given by [10].

3) Accuracy of Algorithm 2: In this section, we verify the accuracy of Algorithm 2 through simulations.

Different from Section VII-B.1, we will simulate a Nakagami-m fading channel with an arbitrary correlation coefficient matrix $\Sigma$, which is more general than the exponential-correlation matrix $\Sigma$ in (29).

One can specify the correlation structure of a Nakagami-m fading channel by its power spectral density denoted by $S(j \omega)$, which is Fourier transform of the auto-correlation function of the channel gain process. We can use factorization to obtain $H(j \omega)$ such that $|H(j \omega)|^{2}=S(j \omega)$, where $|\cdot|^{2}$ is squared magnitude. Then we design a digital filter with frequency response $H(j \omega)$. Given i.i.d. Gaussian random variables $\left\{g_{k, l}\left(k=1, \cdots, N_{s}\right)\right\}$ as the input of the digital filter, the output of the digital filter is a Gaussian random process $\left\{Y_{k, l}\left(k=1, \cdots, N_{s}\right)\right\}$ with power spectral density $S(j \omega)$. Since $l=1, \cdots, 2 m$, we need $2 m$ digital filters, all of which have the same frequency response $H(j \omega)$. In our simulation, we let $H(j \omega)$ be raised cosine with a maximum frequency $f_{m}$, which is also maximum Doppler frequency.

We use the following method to generate a Nakagami-m fading process with power spectral density $S(j \omega)$ : for a given $k(k>1)$, we first generate i.i.d. Gaussian random variables $\left\{g_{k, l}(l=1, \cdots, 2 m)\right\}$; then the $2 m$ digital filters with frequency response $H(j \omega)$ take $\left\{g_{k, l}(l=1, \cdots, 2 m)\right\}$ as their input, respectively, and output $\left\{Y_{k, l}(l=1, \cdots, 2 m)\right\}$ in parallel, i.e., $Y_{k, l}$ is the output of Filter $l$, given its 
input $g_{k, l}$; then use (27) to generate $X_{k}$; then increase $k$ by one and repeat this process. By doing so, we obtain $X_{1}, \cdots, X_{N_{s}}$. Since we recursively generate the channel gain $X_{k}$, the total number of samples $N_{s}$ can be arbitrarily large, which is an advantage of our method.

Given $N_{e}$ measurements of the channel gain, i.e., $X_{1}, X_{2}, \cdots, X_{N_{e}}$ (where $N_{e}=10^{7}$ ), we run Algorithm 2 to estimate $\Sigma$ of dimension $N_{t} \times N_{t}$ and the effective capacity $\widehat{\alpha}_{e}(u)$. To obtain the measured EC function $\alpha_{s}(u)$, we collect measurements from the simulated queueing system shown in Fig. 2, and calculate $\alpha_{s}(u)$ via (11) through (15).

Figs. 7 and 8 show the estimated EC function $\widehat{\alpha}_{e}(u)$ (obtained via Algorithm 2) and the measured EC function $\alpha_{s}(u)$ obtained from the simulation, under different SNR, different values of $m$, and different Doppler frequencies. It is observed that the curves for $\widehat{\alpha}_{e}(u)$ and $\alpha_{s}(u)$ are well matched. This indicates that Algorithm 2 provides an accurate estimate of the effective capacity of an arbitrary correlated Nakagami$\mathrm{m}$ fading channel. The simulation results demonstrate that the estimation-based Algorithm 2 is accurate enough so that one can apply the EC technique to the design of practical QoS provisioning mechanisms.

\section{CONCLUSIONS}

In this paper, we derived a closed-form effective capacity formula for a special correlated Nakagami$\mathrm{m}$ fading channel, for which the inverse of the correlation coefficient matrix is tridiagonal. We also developed Green's matrix based Algorithm 1 to compute the effective capacity for a general correlated Nakagami-m fading channel, given the correlation coefficient matrix of the channel. To verify the accuracy of Algorithm 1 and our EC formula via simulation, we developed a methodology, which allows us to analytically obtain the effective capacity (given the joint pdf of the fading channel) while being able to simulate the corresponding channel gain process. Simulation results showed that Algorithm 1 and our EC formula are accurate. In practice, we cannot directly use Algorithm 1 to compute the effective capacity since the correlation coefficient matrix $\Sigma$ of a Nakagami-m fading channel is not known a priori. To address this, we proposed estimation-based Algorithm 2 to estimate the EC function, given channel gain measurements. Simulation results showed Algorithm 2 is accurate, indicating the excellent practicality of our algorithm.

The implication of this work is significant. Due to lack of closed-form EC formula for the general Nakagami-m fading channels, most previous works only focus on i.i.d. Rayleigh fading channels. Now, we have derived the general EC formula and an algorithm for arbitrary correlated Nakagami-m fading channels and verified their accuracy; this allows researchers to use the EC formula and our algorithm to analyze and design communication systems under arbitrary correlated Nakagami-m fading channels, 
instead of i.i.d. Rayleigh fading channels only. More importantly, our estimation-based algorithm is shown to be accurate, which significantly facilitates the application of the EC theory to the design of practical QoS provisioning mechanisms in wireless networks.

\section{ACKNOWLEDGMENT}

This work was supported in part by the US National Science Foundation under grant CNS-0643731, the US Office of Naval Research under grant N000140810873, NSFC/RGC Joint Research Scheme No. 60831160524 and the open research fund of National Mobile Communications Research Laboratory, Southeast University, China.

\section{APPENDIX}

\section{A. Proof of Proposition 1}

Proof: 1) According to (16), it is easy to see that $X_{k}^{2}(k=1, \cdots, N)$ are random variables having the same Chi-square distribution with $2 m$ degrees of freedom and mean $2 m \sigma^{2}$. Hence, $X_{1}^{2}, \cdots, X_{N}^{2}$ are identically distributed. Therefore, $X_{1}, \cdots, X_{N}$ are also identically distributed; their distribution is Chidistribution with mean $\mu_{X}$ and variance $\nu_{X}^{2}$.

2) Since random variables $X_{k}(k=1,2, \cdots, N)$ are identically distributed with mean $\mu_{X}$ and variance $\nu_{X}^{2}$, we have

$$
E\left[X_{k}^{2}\right]=\mu_{X}^{2}+\nu_{X}^{2}
$$

The covariance $\operatorname{Cov}\left(X_{i}^{2}, X_{j}^{2}\right)$ between random variables $X_{i}^{2}$ and $X_{j}^{2}$ is given by

$$
\begin{aligned}
\operatorname{Cov}\left(X_{i}^{2}, X_{j}^{2}\right) & =E\left[\left(X_{i}^{2}-E\left[X_{i}^{2}\right]\right)\left(X_{j}^{2}-E\left[X_{j}^{2}\right]\right)\right] \\
& \stackrel{(a)}{=} E\left[X_{i}^{2} X_{j}^{2}\right]-\left(\mu_{X}^{2}+\nu_{X}^{2}\right)^{2} \\
& =R\left(X_{i}^{2}, X_{j}^{2}\right)-\left(\mu_{X}^{2}+\nu_{X}^{2}\right)^{2}
\end{aligned}
$$

where (a) is due to (31).

3) According to (16), for $k=1,2, \cdots, N$, we have

$$
\begin{aligned}
E\left[X_{k}^{2}\right] & =E\left[Y_{k, 1}^{2}\right]+E\left[Y_{k, 2}^{2}\right]+\cdots+E\left[Y_{k, 2 m}^{2}\right] \\
& =2 m \sigma^{2}
\end{aligned}
$$

Combining (31) with (33), we have

$$
\mu_{X}^{2}+\nu_{X}^{2}=2 m \sigma^{2}
$$


Next, we derive correlation $R\left(X_{i}^{2}, X_{j}^{2}\right)$ between random variables $X_{i}^{2}$ and $X_{j}^{2}$ as below,

$$
\begin{aligned}
R\left(X_{i}^{2}, X_{j}^{2}\right) & =E\left[X_{i}^{2} X_{j}^{2}\right] \\
& \stackrel{(a)}{=} E\left[\left(Y_{i, 1}^{2}+Y_{i, 2}^{2}+\cdots+Y_{i, 2 m}^{2}\right) \times\left(Y_{j, 1}^{2}+Y_{j, 2}^{2}+\cdots+Y_{j, 2 m}^{2}\right)\right] \\
& =E\left[\sum_{l=1}^{2 m} Y_{i, l}^{2} Y_{j, l}^{2}+\sum_{l_{1}=1}^{2 m} \sum_{l_{2=1}}^{2 m} Y_{i, l_{1}}^{2} Y_{j, l_{2}}^{2}\right] \\
& \stackrel{(b)}{=} \sum_{l=1}^{2 m} E\left[Y_{i, l}^{2} Y_{j, l}^{2}\right]+\sum_{l_{1}=1}^{2 m} \sum_{\substack{l_{2}=1 \\
l_{2} \neq l_{1}}}^{2 m} E\left[Y_{i, l_{1}}^{2} Y_{j, l_{2}}^{2}\right] \\
& \stackrel{(c)}{=} \sum_{l=1}^{2 m} E\left[Y_{i, l}^{2} Y_{j, l}^{2}\right]+\sum_{l_{1}=1}^{2 m} \sum_{\substack{l_{2}=1 \\
l_{2} \neq l_{1}}}^{2 m} E\left[Y_{i, l_{1}}^{2}\right] E\left[Y_{j, l_{2}}^{2}\right] \\
& \stackrel{(d)}{=} 2 m E\left[Y_{i, l}^{2} Y_{j, l}^{2}\right]+2 m(2 m-1) \sigma^{4}
\end{aligned}
$$

where (a) is from (16); (b) is because for any $i(i=1, \cdots, N), Y_{i, 1}, Y_{i, 2}, \cdots, Y_{i, 2 m}$ are independent, and the column vectors $\left\{\left[Y_{1, l}, Y_{2, l}, \cdots, Y_{N, l}\right]^{T},(l=1, \cdots, 2 m)\right\}$ are independent; (c) $E\left[Y_{i, l_{1}}^{2} Y_{j, l_{2}}^{2}\right]=$ $E\left[Y_{i, l_{1}}^{2}\right] E\left[Y_{j, l_{2}}^{2}\right]$ holds because the column vectors $\left\{\left[Y_{1, l}, Y_{2, l}, \cdots, Y_{N, l}\right]^{T},(l=1, \cdots, 2 m)\right\}$ are independent and hence $Y_{i, l_{1}}$ and $Y_{j, l_{2}}\left(i \neq j\right.$ and $\left.l_{1} \neq l_{2}\right)$ are independent; and (d) is because the column vectors $\left\{\left[Y_{1, l}, Y_{2, l}, \cdots, Y_{N, l}\right]^{T},(l=1, \cdots, 2 m)\right\}$ are i.i.d. and $E\left[Y_{k, l}^{2}\right]=\sigma^{2}(\forall k, \forall l)$.

Since it is known that if $X$ and $Y$ are identically distributed Gaussian random variables with zero mean and variance $\sigma^{2}$, and their correlation coefficient is $\rho$, then $E\left[X^{2} Y^{2}\right]=\left(2 \rho^{2}+1\right) \sigma^{4}$ holds, we obtain

$$
E\left[Y_{i, l}^{2} Y_{j, l}^{2}\right]=\left(2 \Sigma_{i, j}^{2}+1\right) \sigma^{4}
$$

Substituting (36) into (35), we have

$$
\begin{aligned}
R\left(X_{i}^{2}, X_{j}^{2}\right) & =2 m\left(2 \Sigma_{i, j}^{2}+1\right) \sigma^{4}+2 m(2 m-1) \sigma^{4} \\
& \stackrel{(a)}{=}\left(\mu_{X}^{2}+\nu_{X}^{2}\right)^{2}\left(\Sigma_{i, j}^{2}+m\right) / m
\end{aligned}
$$

where (a) is due to substituting $\sigma^{4}$ by square of (34). Substituting (37) into (32), we obtain

$$
\operatorname{Cov}\left(X_{i}^{2}, X_{j}^{2}\right)=\left(\mu_{X}^{2}+\nu_{X}^{2}\right)^{2} \times \Sigma_{i, j}^{2} / m
$$

From (38), we have

$$
\Sigma_{i, j}=\frac{\sqrt{m \times \operatorname{Cov}\left(X_{i}^{2}, X_{j}^{2}\right)}}{\mu_{X}^{2}+\nu_{X}^{2}}
$$




\section{REFERENCES}

[1] J. Le Boudec and P. Thiran, Network calculus: a theory of deterministic queuing systems for the internet. Springer Verlag, 2001.

[2] R. Cruz, "A calculus for network delay. I. Network elements in isolation," IEEE transactions on information theory, vol. 37, no. 1, pp. 114-131, 1991.

[3] J. Kim and M. Krunz, "Bandwidth allocation in wireless networks with guaranteed packet-loss performance," IEEE/ACM Transactions on networking, vol. 8 , no. 3, p. 337, 2000.

[4] _ - "Fluid analysis of delay and packet discard performance for QoS support in wireless networks," IEEE Journal on Selected Areas in Communications, vol. 19, pp. 384-395, 2001.

[5] Y. Kim and S. Li, "Capturing important statistics of a fading/shadowing channel fornetwork performance analysis," IEEE Journal on Selected Areas in Communications, vol. 17, no. 5, pp. 888-901, 1999.

[6] M. Schwartz, Broadband integrated networks. Prentice-Hall, Inc. Upper Saddle River, NJ, USA, 1996.

[7] A. Dembo and O. Zeitouni, Large deviations techniques and applications. Boston: Jones and Bartlett, 1992.

[8] A. Weiss and A. Shwartz, "Large Deviations for Performance Analysis," 1995.

[9] D. Wu, "Providing quality of service guarantees in wireless networks," Ph.D. Dissertation, Dept. of Electrical \& Computer Engineering, Carnegie Mellon University, Aug. 2003. [Online]. Available: http://www.wu.ece.ufl.edu/mypapers/Thesis.pdf.

[10] D. Wu and R. Negi, "Effective capacity: a wireless link model for support of quality of service," IEEE Transactions on wireless communications, vol. 2, no. 4, pp. 630-643, 2003.

[11] D. Wu, "QoS provisioning in wireless networks," Wireless Communications and Mobile Computing, vol. 5, no. 8, 2005.

[12] X. Zhang, J. Tang, H. Chen, S. Ci, and M. Guizani, "Cross-layer-based modeling for quality of service guarantees in mobile wireless networks," IEEE Communications Magazine, vol. 44, no. 1, pp. 100-106, 2006.

[13] J. Tang and X. Zhang, "Quality-of-service driven power and rate adaptation for multichannel communications over wireless links," IEEE Transactions on Wireless Communications, vol. 6, no. 12, p. 4349, 2007.

[14] X. Zhang, J. Tang, H. Chen, S. Ci, and M. Guizani, "Cross-layer-based modeling for quality of service guarantees in mobile wireless networks,” IEEE Communications Magazine, vol. 44, no. 1, pp. 100-106, 2006.

[15] J. Tang and X. Zhang, "Cross-layer resource allocation over wireless relay networks for quality of service provisioning," IEEE Journal on Selected Areas in Communications, vol. 25, no. 4, pp. 645-656, 2007.

[16] — - "Cross-layer-model based adaptive resource allocation for statistical QoS guarantees in mobile wireless networks," IEEE Transactions on Wireless Communications, vol. 7, no. 6, pp. 2318-2328, 2008.

[17] Y. Chen, J. Chen, and Y. Yang, "Multi-hop delay performance in wireless mesh networks," Mob. Netw. Appl., vol. 13, no. 1-2, pp. $160-168,2008$.

[18] W. Kumwilaisak, Y. Hou, Q. Zhang, W. Zhu, C. Kuo, and Y. Zhang, "A cross-layer quality-of-service mapping architecture for video delivery in wireless networks," IEEE Journal on Selected Areas in Communications, vol. 21, no. 10, pp. 1685-1698, 2003.

[19] L. Liu, P. Parag, J. Tang, W. Chen, and J. Chamberland, "Resource allocation and quality of service evaluation for wireless communication systems using fluid models," IEEE Transactions on Information Theory, vol. 53, no. 5, pp. 1767-1777, 2007.

[20] Q. Wang, D. Wu, and P. Fan, "Effective Capacity of a Correlated Rayleigh Fading Channel," Wireless Communications and Mobile Computing, 2010.

[21] C. Chang and J. Thomas, "Effective bandwidth in high-speed digital networks," IEEE Journal on Selected areas in Communications, vol. 13, no. 6, pp. 1091-1100, 1995.

[22] M. Nakagami, "The m-distribution: A general formula of intensity distribution of rapid fading, Statistical Methods in Radio Wave Propagation," Elmsford, NY: Pergamon, 1960.

[23] G. Karagiannidis, D. Zogas, and S. Kotsopoulos, “An efficient approach to multivariate Nakagami-m distribution using Green's matrix approximation," IEEE Transactions on Wireless Communications, vol. 2, no. 5, pp. 883-889, 2003.

[24] R. Nabben, “On Green's matrices of trees,” SIAM Journal on Matrix Analysis and Applications, vol. 22, no. 4, pp. 1014-1026, 2001.

[25] L. Blumenson and K. Miller, "Properties of generalized Rayleigh distributions," The Annals of Mathematical Statistics, pp. 903-910, 1963.

[26] K. Levenberg, "A Method for the Solution of Certain Non-Linear Problems in Least Squares," The Quarterly of Applied Mathematics, vol. 2, pp. 164-168, 1944.

[27] H. Holma and A. Toskala, WCDMA for UMTS: Radio access for third generation mobile communications. John Wiley \& Sons, Inc. New York, NY, USA, 2000.

[28] Q. Zhang, "A decomposition technique for efficient generation of correlated Nakagami fading channels," IEEE Journal on Selected Areas in Communications, vol. 18, no. 11, pp. 2385-2392, 2000. 


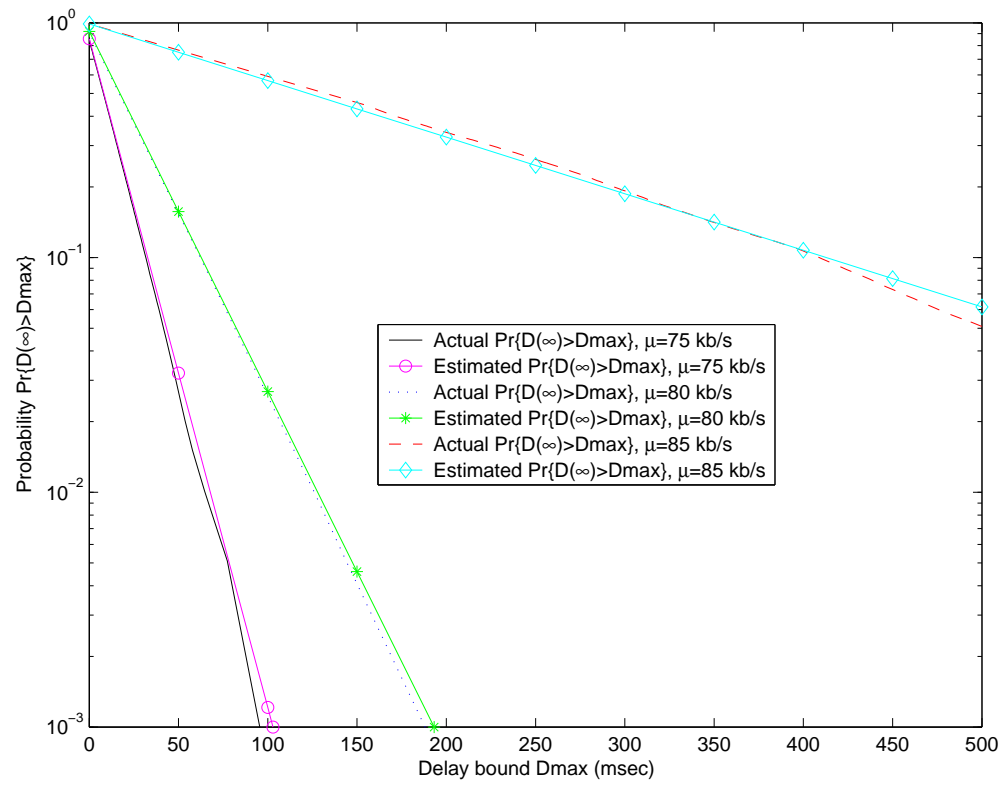

Fig. 1. Estimated $\operatorname{Pr}\left\{D(\infty) \geq D_{\max }\right\}$ from large deviations techniques for the small delay regime.

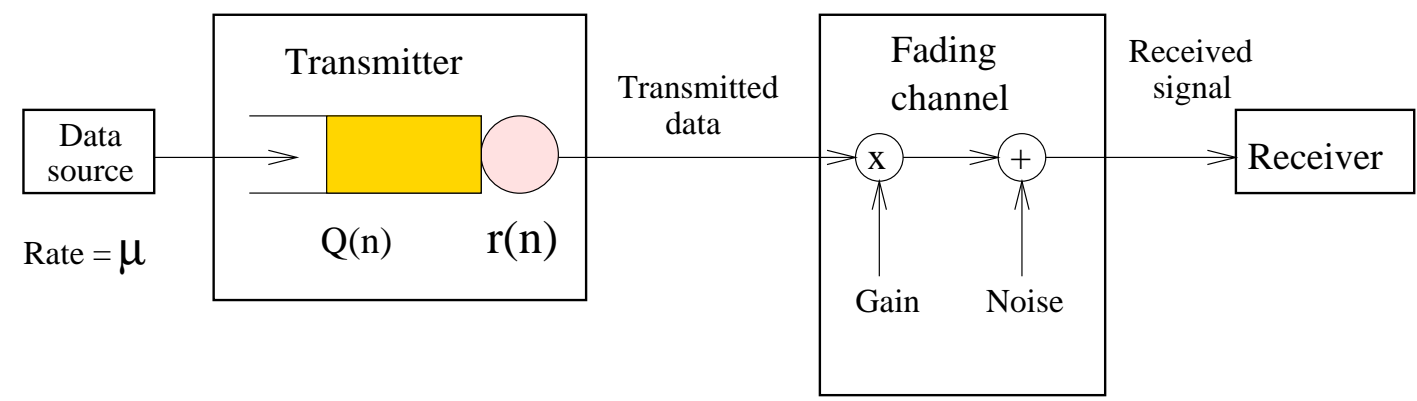

Fig. 2. Queueing system model.

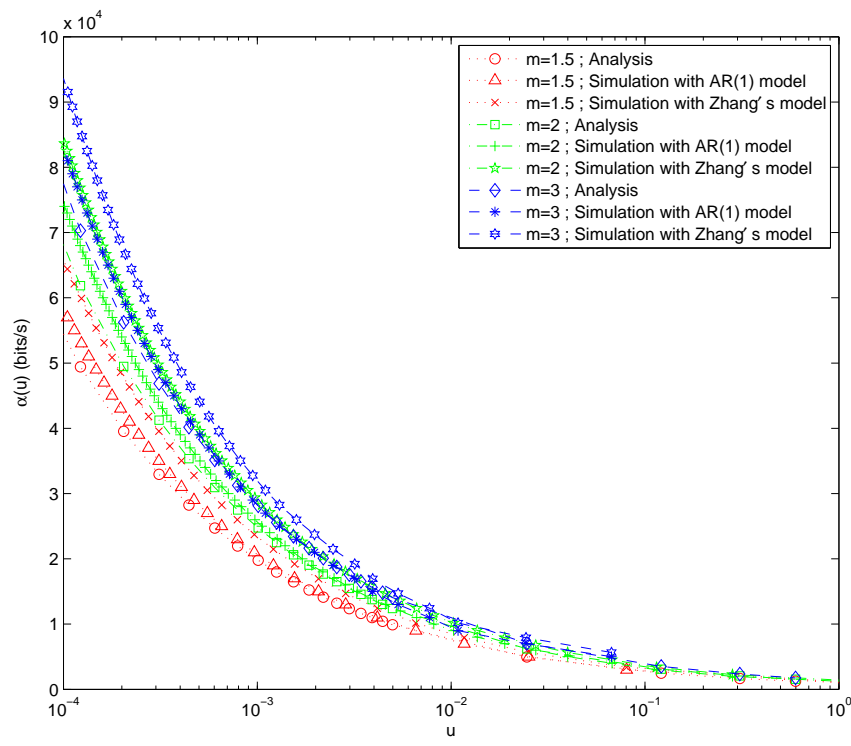

Fig. 3. $\alpha^{(d)}(u)$ (analysis) vs. $\alpha_{s}(u)$ and $\alpha_{s}^{Z}(u)$ (simulation) for Doppler frequency $f_{m}=15 \mathrm{~Hz}$. 


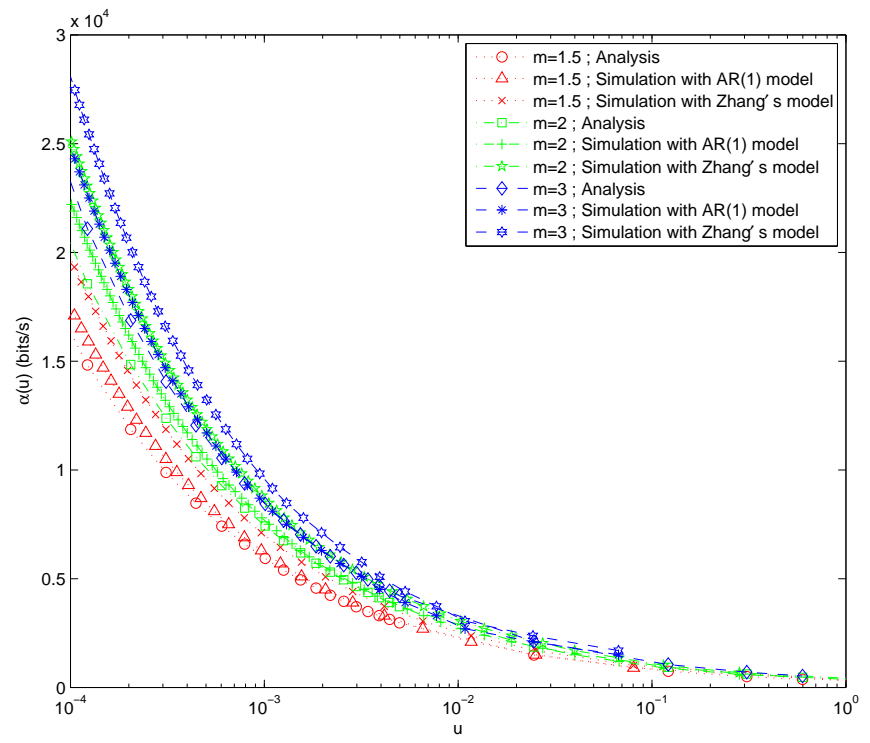

Fig. 4. $\alpha^{(d)}(u)$ (analysis) vs. $\alpha_{s}(u)$ and $\alpha_{s}^{Z}(u)$ (simulation) for $f_{m}=100 \mathrm{~Hz}$.

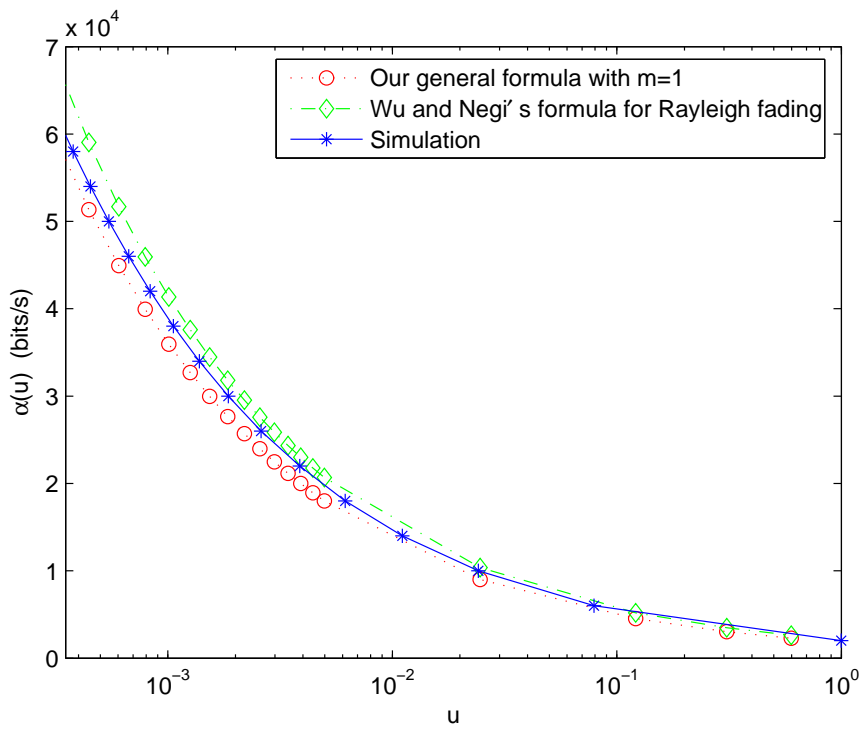

Fig. 5. $\quad \alpha^{(d)}(u)$ (from our general formula), $\alpha(u)$ (from Wu and Negi's formula), and $\alpha_{s}\left(u\right.$ ) (simulation) for $f_{m}=15 \mathrm{~Hz}$. 


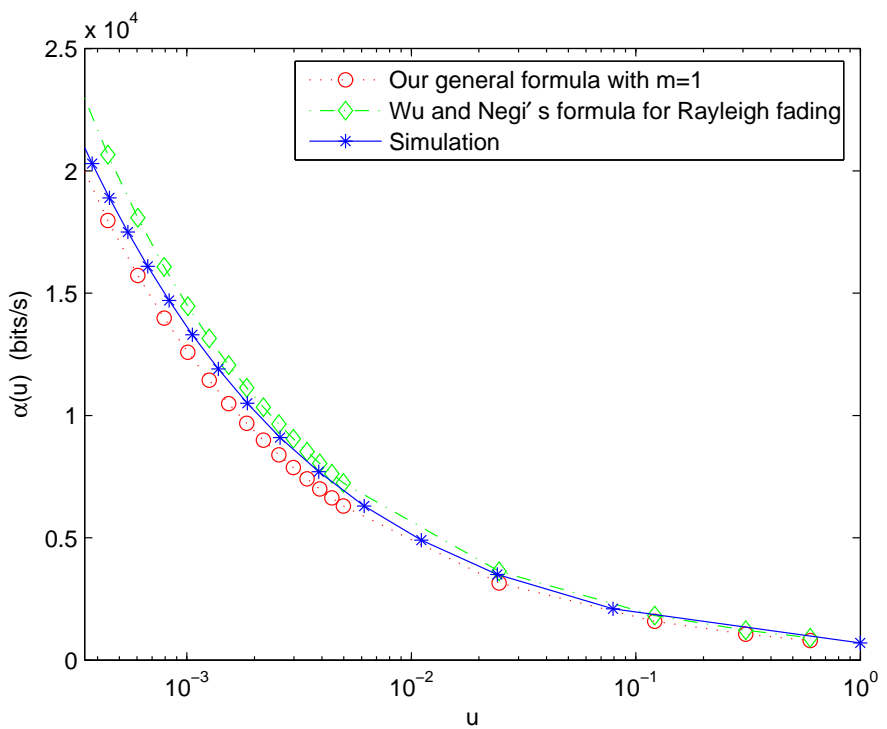

Fig. 6. $\quad \alpha^{(d)}(u)$ (from our general formula), $\alpha(u)$ (from Wu and Negi's formula), and $\alpha_{s}(u)$ (simulation) for $f_{m}=100 \mathrm{~Hz}$.

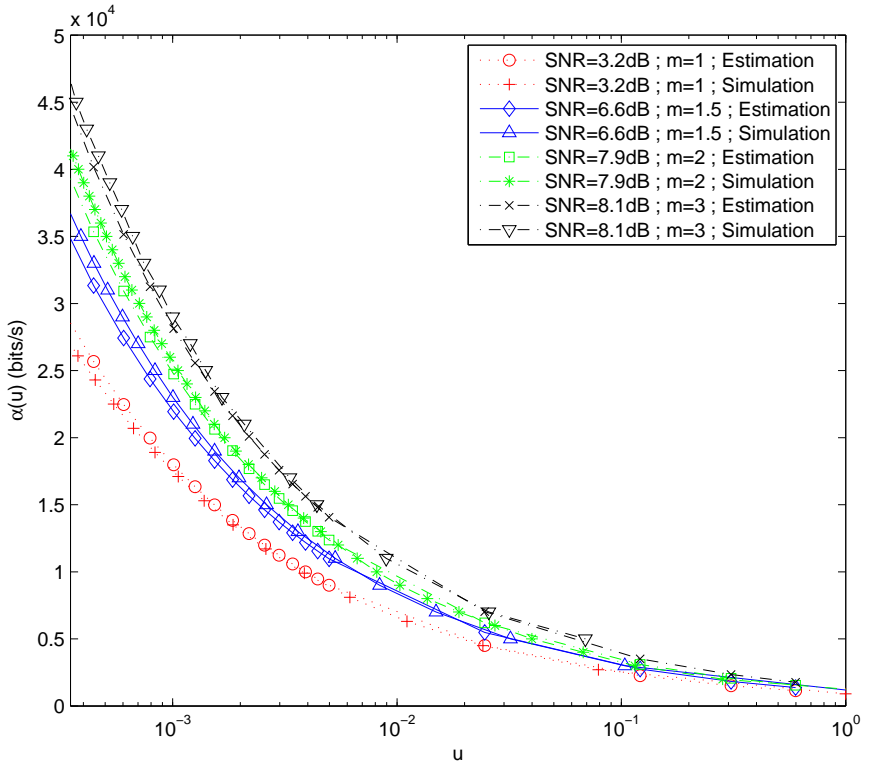

Fig. 7. $\widehat{\alpha}_{e}(u)$ (estimation) vs. $\alpha_{s}(u)$ (simulation) for $f_{m}=15 \mathrm{~Hz}$. 


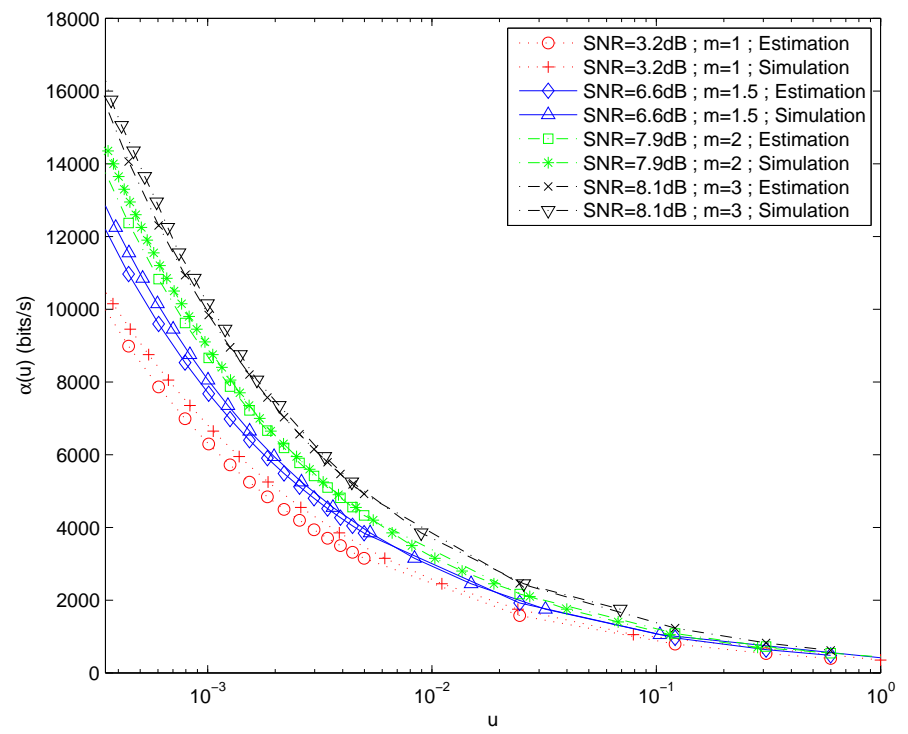

Fig. 8. $\widehat{\alpha}_{e}(u)$ (estimation) vs. $\alpha_{s}(u)$ (simulation) for $f_{m}=100 \mathrm{~Hz}$. 